\title{
Aptamer-antibody sandwich assay for cytochrome c employing a MWCNT platform and the electrochemical impedance technique
}

\author{
Cristina Ocaña ${ }^{1}$, Sonja Lukic ${ }^{2}$ and Manel del Valle ${ }^{1 *}$ \\ ${ }^{1}$ Sensors and Biosensors Group, Department of Chemistry, Universitat Autònoma de \\ Barcelona, Edifici Cn, 08193 Bellaterra, Barcelona, SPAIN \\ ${ }^{2}$ Institute of Analytical Chemistry, Chemo- and Biosensors, University of Regensburg, \\ 93053 Regensburg, GERMANY
}

\begin{abstract}
In this work, we report a sensitive aptamer-antibody assay for cytochrome c (Cytc) detection using electrochemical impedance technique. The biosensor design involves the use of 4-amino benzoic acid for the oriented immobilization of aminated aptamers onto multi-walled carbon nanotube screen-printed electrode surfaces through an electrochemical grafting technique; electrochemical impedance measurements were performed in a solution containing the redox marker ferrocyanide/ferricyanide. The change in interfacial charge transfer resistance (Rct) experimented by the redox marker, was recorded to confirm aptamer complex formation with target protein, (Cytc), in a label-free first stage. A biotinylated anti-cytochrome c antibody (AbCytc) was then used in a sandwich approach. The addition of strepAuNPs and silver enhancement treatment led to a further increment of Rct thus obtaining significant signal amplification. Under the optimized working conditions, an extremely low detection limit of less than 2pM was obtained. Cross-reactivity was evaluated against other proteins present in serum (fibrinogen, BSA, immunoglobulin G) and the obtained results demonstrated an improved selectivity. In order to visualize the presence of gold nanoparticles, gold enhancement treatment was applied to electrodes already modified with the nanoparticle-sandwich conjugate, allowing direct observation by scanning electron microscopy (SEM).
\end{abstract}

Keywords: Aptamer, sandwich, cytochrome c, SEM, gold-nanoparticles

Electrochemical Impedance Spectroscopy.

*E-mail: manel.delvalle@uab.cat; tel: +34 93 5813235; fax: +34 935812477 


\section{Introduction}

Cytochrome c (Cytc) is a heme containing metalloprotein located in the intermembrane space of mitochondria. It plays a central role in electron transport chain and also it is an intermediate in apoptosis. When mitochondria are injured under pathological conditions, Cytc is released into the cytosol of the cell. This translocation of Cytc from mitochondria to cytosol is a decisive event in the activation of intracellular signaling; it results in a cascade of caspase activation and guides to program cell apoptosis. For this reason, the quantification of Cytc could be of great importance in clinical diagnosis and therapeutic research [1].

Aptamers are oligonucleotides (DNA or RNA) that possess properties comparable to those of protein monoclonal antibodies, and thus are clear alternatives to long established antibody-based diagnostic or other biotechnological products for research [2,3], therapy [4,5] and diagnostics [6,7]. This kind of functional nucleic acids can fold into complex three dimensional shapes forming binding pockets and cavities able to the specific recognition and high affinity binding of any given molecular target, from metal ions and small chemicals structures to large proteins and higher order proteins complexes, even whole cells, viruses or parasites [8]. Aptamers are generated by an in vitro selection process called SELEX (Systematic Evolution of Ligands by Exponential Enrichment), which was first reported in 1990 [9,10]. This method has permitted the identification of unique DNA/RNA molecules, from large sets of random sequence oligomers (DNA or RNA libraries), which bind to specific target molecule with very high specificity and affinity [11]. Due to these interesting characteristics, aptamers have been increasingly used in biosensing in the recent years [12-15].

Biosensors can be classified depending on the technique employed for transduction, into optical [16,17], piezoelectric [18,19] and electrochemical types $[20,21]$. In recent years, among the different electrochemical techniques available, electrochemical impedance spectroscopy (EIS) [22] has been used in numerous studies $[23,24]$. This technique is very sensitive to changes in the interfacial properties of the modified electrodes caused by biorecognition events at the electrode surface $[25,26]$. For this reason, EIS is becoming an attractive electrochemical technique for numerous applications such as immunosensing [27], enzyme activity determination [28], genosensing [29,30], studies of corrosion [31] and or other surface phenomena [32]. 
Signal amplification based on biofunctional nanomaterials has recently attracted considerable attention due to the need for ultrasensitive bioassays. Among nanomaterials, gold nanoparticles have been widely used thanks to their excellent properties, such as high biocompatibility, distinctive size-related electronic and optical behaviour, high electrical conductivity and high catalytic activity [33]. For example, Deng et al. [34] used AuNPs stabilized with sodium dodecylsulfate to amplify the impedimetric signal for the detection of thrombin, Zheng et al. [35] used network-like thiocyanuric acid/gold nanoparticles to amplify the signal for the detection of thrombin, etc.

In this work, we report a sensitive impedimetric aptamer-antibody sandwich assay for Cytc detection using a highly specific amplification strategy with the use of streptavidin gold nanoparticles and silver enhancement treatment. The employed transducer consisted of a multi-walled carbon nanotube (MWCNT) screen-printed electrode, its surface allowing the immobilization of cytochrome c aptamer (AptCytc) by covalent bond via prior electrochemical grafting. As a transducer material, MWCNTs are used for promoting electron-transfer between the electroactive species and electrode and provide a novel method for fabricating biosensors. The change of interfacial charge transfer resistance (Rct) experimented by the redox marker, was recorded to confirm the aptamer complex formation with target protein, cytochrome $\mathrm{c}$ (Cytc). After that, a biotinylated anti-cytochrome c antibody (AbCytc) is used to form the sandwich. The addition of strep-AuNPs and silver enhancement treatment led to a further increment of Rct and the subsequent achievement of significant signal amplification, high sensitivity and improvement of selectivity.

\section{Experimental}

\section{Reagents and solutions}

Potassium dihydrogen phosphate, potassium ferricyanide $\mathrm{K}_{3}\left[\mathrm{Fe}(\mathrm{CN})_{6}\right]$, potassium ferrocyanide $\mathrm{K}_{4}\left[\mathrm{Fe}(\mathrm{CN})_{6}\right]$, sodium monophosphate, 4-aminobenzoic acid (ABA), sodium nitrite, $N$-(3-dimethylaminopropyl)- $N$ '-ethylcarbodiimide hydrochloride (EDC), gold (III) chloride solution $\left(\mathrm{HAuCl}_{4}\right), \quad N$-hydroxysuccinimide (NHS), streptavidin gold nanoparticles, fibrinogen, immunoglobulin $G$ and the target protein 
cytochrome c (Cytc), were purchased from Sigma (St. Louis, MO, USA). Poly(ethylene glycol) 1000 (PEG), sodium chloride, hydroxylamine hydrochloride $\left(\mathrm{NH}_{2} \mathrm{OH} \cdot \mathrm{HCl}\right)$ and potassium chloride were purchased from Fluka (Buchs, Switzerland). Polyclonal biotinylated anti-cytochrome c antibody (AbCytc) was purchased from BioLegend (San Diego, California). LI silver enhancement kit was obtained from Nanoprobes (Yaphank, New York). All reagents were analytical reagent grade. The aptamer used in this study was synthetized by TIB-MOLBIOL (Berlin, Germany). Stock solutions of aptamers were diluted with sterilized and deionised water, separated into fractions and stored at $-20{ }^{\circ} \mathrm{C}$ until required. Aptamer solutions were prepared in PBS buffer $\mathrm{pH} 7$ from stock solutions. A well-known aptamer for thrombin (AptThr) was used for negative control purposes. Their base sequences were:

AptCytc:

5'-NH 2 -AGTGTGAAATATCTAAACTAAATGTGGAGGGTGGG

ACGGGAAGAAGTTTATTTTCACACT-3'

AptThr:

5'-AGTCCGTGGTAGGGCAGGTTGGGGTGACT-Biotin-3'

All solutions were made up using MilliQ water from MilliQ System (Millipore, Billerica, MA, USA). The buffers employed were: PBS (187 mM NaCl, $2.7 \mathrm{mM} \mathrm{KCl}$, $8.1 \mathrm{mM} \mathrm{Na}_{2} \mathrm{HPO}_{4} \cdot 2 \mathrm{H}_{2} \mathrm{O}, 1.76 \mathrm{mM} \quad \mathrm{KH}_{2} \mathrm{PO}_{4}, \mathrm{pH}$ 7.0) and triethylammonium bicarbonate $0.6 \mathrm{M}$.

\section{Biosensing Protocol}

The steps of the experimental protocol for Cytc analysis, described in detail below, are represented in Figure 1.

$<$ FIGURE 1>

\section{Aptamer immobilization}

MWCNT screen-printed electrodes were modified with aminobenzoic acid by means of a one step procedure. Firstly, $30 \mathrm{mg}$ of ABA were dissolved in $3 \mathrm{~mL}$ of $1 \mathrm{M}$ $\mathrm{HCl}$ and cooled with ice. Then, the diazonium salt was prepared by adding $570 \mu \mathrm{L}$ of 2 $\mathrm{mM} \mathrm{NaNO} \mathrm{N}_{2}$ aqueous solution dropwise to the 4-aminobenzoic acid solution, with 
constant stirring. The electrode was immersed in this solution, and 10 successive voltammetric cycles ranging between 0.0 and $-1.0 \mathrm{~V}\left(v=200 \mathrm{mV} \cdot \mathrm{s}^{-1}\right)$ were performed [27], generating a carbon-carbon bond and eliminating the azonium group. The modified electrodes (benzoic acid modified) were washed thoroughly with water and methanol and dried at room temperature. Finally, $60 \mu \mathrm{L}$ of aptamer solution with $1 \mathrm{mg}$ of EDC and $0.5 \mathrm{mg}$ of NHS was placed on the modified electrode and left to react for $12 \mathrm{~h}$, with the goal of covalent immobilization of the aptamer through the amide formation. This step was followed by two 10 min washing steps with PBS buffer solution.

\section{Blocking step}

To minimize any possible nonspecific adsorption of secondary species, $60 \mu 1$ of PEG were dropped onto the electrodes and left to incubate during $15 \mathrm{~min}$. This was followed by two washing steps using PBS buffer solution for $10 \mathrm{~min}$.

\section{Cytochrome c detection}

$60 \mu \mathrm{l}$ of a solution with the desired concentration of Cytc were dropped onto the electrodes. The incubation took place for $15 \mathrm{~min}$. Then, the biosensors were washed twice with PBS buffer solution for $10 \mathrm{~min}$.

\section{Sandwich formation}

In order to achieve the aptamer sandwich formation, the electrodes were dropped with $60 \mu \mathrm{l}$ of AbCytc, from a 1/500 dilution of the stock solution in PBS buffer. The incubation took place for $15 \mathrm{~min}$. This was followed by two washing steps using PBS buffer solution for $10 \mathrm{~min}$.

\section{Addition of strep-AuNPs}

$60 \mu \mathrm{L}$ of strep-AuNPs, from a 1/100 dilution of the stock solution in PBS buffer were dropped onto the electrodes [36]. This step was followed by two gentle washing steps in PBS buffer for $10 \mathrm{~min}$ at $25^{\circ} \mathrm{C}$. Negative controls were performed for the strepAuNPs addition step using AptThr as an aptamer without affinity. 
$20 \mu 1$ of a solution obtained by the combination of $10 \mu \mathrm{l}$ of enhancer and $10 \mu \mathrm{l}$ of initiator (commercial solutions) were deposited onto the electrode surface and left for 7 minutes to facilitate the reaction [36]. After the catalytic silver reduction, the electrodes were thoroughly washed with deionized water to stop the reaction. The silver enhancing solution was prepared immediately before each use. For silver enhancement treatment, the negative control used was a biotinylated AptThr as aptamer without affinity.

\section{Gold enhancement of strep-AuNPs}

The MWCNT screen-printed electrodes modified with sandwich and strepAuNPs were immersed in a solution containing a mixture of $0.01 \% \mathrm{HAuCl}_{4}$ and 0.4 $\mathrm{mM} \mathrm{NH} \mathrm{NH}_{2} \mathrm{OH} \cdot \mathrm{HCl}(\mathrm{pH} 6.0)$ for $2 \mathrm{~min}$ at $25^{\circ} \mathrm{C}$, rinsed, and then treated for 2 additional min. In order to prevent the non-specific background of fine gold particles, the electrodes were rinsed with a solution of $0.6 \mathrm{M}$ triethylammonium bicarbonate buffer after each amplification. Solutions were freshly prepared in a lightproof container before each use.

Different selectivity experiments were carried out to verify selectivity characteristics of the assay with potentially interfering proteins instead of Cytc.

\section{Equipment}

AC impedance measurements were performed with the aid of an Autolab PGStat 20 (Metrohm Autolab B.V, Utrecht, The Netherlands). FRA (Metrohm Autolab) software was used for data acquisition and control of the experiments. A three electrode configuration was used to perform the impedance measurements: a platinum-ring auxiliary electrode (Crison 52-67 1, Barcelona, Spain), a $\mathrm{Ag} / \mathrm{AgCl}$ reference electrode and the MWCNT-screen printed electrode as the working electrode (Dropsens, Oviedo, Spain). A scanning electron microscope (SEM) (Merlin, Zeiss, Germany) was used to visualize gold enhanced strep-AuNPs on the electrode surface.

\section{EIS detection}

Impedance experiments were performed at an applied potential of $0.17 \mathrm{~V}$ (vs. $\mathrm{Ag} / \mathrm{AgCl}$ reference electrode), with a range of frequency of $50 \mathrm{KHz}-0.05 \mathrm{~Hz}$, an $\mathrm{AC}$ amplitude of $10 \mathrm{mV}$ and a sampling rate of 10 points per decade above $66 \mathrm{~Hz}$ and 5 
points per decade at the lower range. All measurements were performed in PBS buffer containing $0.01 \mathrm{M} \mathrm{K}_{3}\left[\mathrm{Fe}(\mathrm{CN})_{6}\right] / \mathrm{K}_{4}\left[\mathrm{Fe}(\mathrm{CN})_{6}\right](1: 1)$ mixture, used as a redox marker. The impedance spectra were plotted in the form of complex plane diagrams (Nyquist plots, $-Z_{\text {im }} v s . Z_{\text {re }}$ ) and fitted to a theoretical curve corresponding to the equivalent circuit with $\mathrm{Z}_{\text {view }}$ software (Scribner Associates Inc., USA). The equivalent circuit was formed by one resistor/ capacitor element in series with a resistance; the warburg term was circumvented as the diffusion processes were not relevant in this study. For all performed fittings, the chi-square goodness-of-fit test was thoroughly checked to verify the calculations. In all cases, calculated values for each circuit remained in the range of 0.0003-0.15 much lower than the tabulated value for 50 degrees of freedom (67.505 at 95\% confidence level). The most important parameter in this work is the electron transfer resistance (Rct), which reflects the resistance to charge transfer between the redox probe and the electrode surface. In order to compare the results obtained from the different electrodes used, and to obtain independent and reproducible results, a relative transformation of signals was needed [37]. Thus, the $\Delta_{\text {ratio }}$ value was defined according to the following equations:

$$
\begin{gathered}
\Delta_{\text {ratio }}=\Delta_{\mathrm{s}} / \Delta_{\mathrm{p}} \\
\Delta_{\mathrm{s}}=\operatorname{Rct}_{(\text {AptCytc/Cytc/AbCytc/strep-AuNPs/silverenhancement) }}-\operatorname{Rct}_{\text {(electrode-buffer) }} \\
\Delta_{\mathrm{p}}=\operatorname{Rct}_{(\text {AptCytc) }}-\operatorname{Rct}_{(\text {electrode-buffer })}
\end{gathered}
$$

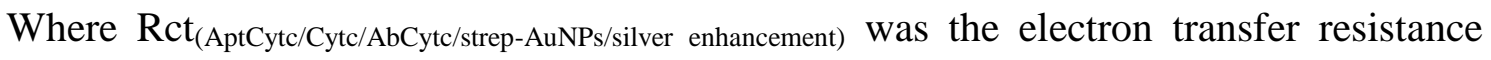
value measured after sandwich formation and silver enhancement treatment; $\operatorname{Rct}_{(\mathrm{AptCytc})}$ was the electron transfer resistance value measured after aptamer immobilization on the

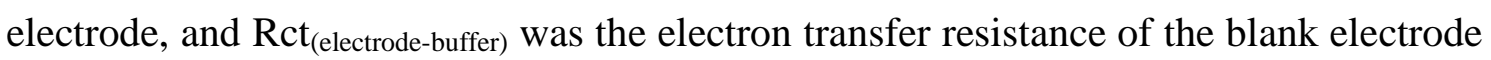
and buffer.

\section{Results and Discussion}

The fundamentals of the developed assay are illustrated in Figure 1. Firstly, MWCNT screen-printed electrodes were modified with amino benzoic acid. Briefly, diazotation of $\mathrm{ABA}$ was performed with sodium nitrite in hydrochloride acid, the resulting 4-carboxybenzenediazonium ion solution was dropped onto the MWCNT 
electrode surface and the potential was cycled as described in Experimental Section. Figure 2a) shows the Nyquist plots obtained by electrochemical impedance spectroscopy. As can be seen, modification of the MWCNT electrode with ABA gave rise to a large increase in the electron transfer resistance as a consequence of the electrostatic repulsion between the redox maker and the negatively charged carboxylate groups. Thereafter, surface-confined carboxyl groups were activated with EDC/NHS to form amide bonds with amino terminated aptamer. Moreover, as it can be observed in Figure $2 b$ ), the Rct value diameter of the semicircle increased after each performed step.

This can be attributed to the augmented difficulty of the redox reaction of $\left[\mathrm{Fe}(\mathrm{CN})_{6}\right]^{3-14}$ to take place at the MWCNT surface, due to the sensor surface alteration by interaction with AptCytc. Two different factors may be taken into account to properly explain this: electrostatic repulsion and sterical hindrance. The former is more significant in the first and second step: when AptCytc is immobilized onto the electrode surface, a second layer is formed, where negatively charged phosphate groups of the DNA skeleton are responsible of the electrical repulsion towards the negatively charged redox marker, thus inhibiting the interfacial electron transfer process and increasing the Rct value. The addition of a target protein, Cytc, and AbCytc to form a complex AptCytc-Cytc and a sandwich respectively, resulted in a decreased increment of the resistance value due to the augmented quantity of negative charges and to the hindrance caused by the formation of a double layer. After the addition of strept-AuNPs we can observe a further increment of charge transfer resistance because of the increased hidrance due to the formed conjugates. In the second amplification step, the silver enhancement treatment [38,39], a significant increment of Rct value was also observed and attributable to the silver deposition on gold.

$<$ FIGURE 2>

\section{Optimization of the experimental concentrations involved in the aptamer- antibody sandwich assay response to Cytc}

All concentrations involved in the analytical performance of the aptansensor for detection of Cytc were optimized by constructing its relative response curve. For this, increasing concentrations of AptCytc and PEG were used to determine the immobilization and surface blocking, respectively, evaluating the changes in the $\Delta \mathrm{p}$. 
Figure 3a) shows the curve of AptCytc immobilization onto the electrode surface. It can be observed that the difference in resistance $(\Delta p)$ increased up to a value. This is due to the physical adsorption of the aptamer onto the electrode surface, which followed a Langmuir isotherm. Embodied by the variation of Rct which increases to reach a saturation value, the optimal concentration was chosen as initial value to reach it. This value corresponded to a concentration of aptamer of $1.5 \mu \mathrm{M}$. Concerning blocking agent, and as shown in Figure 3b), a different behaviour was obtained. The optimal concentration of blocking agent was chosen as $30 \mathrm{mM}$ because it was the concentration point where a small plateau was observed.

\section{$<$ FIGURE 3>}

In addition, in order to obtain the optimal concentration of AbCytc to be used in the biosensing protocol, response was evaluated with increasing concentration of antibody. The optimal concentration was evaluated by the changes in the $\Delta_{\text {ratio }}, 1 / 500$ stock dilution, Figure S1.

\section{Scanning electron microscope examination}

Screen-printed MWCNT electrode surfaces were investigated by SEM after the Gold Enhancement treatment. $\mathrm{HAuCl}_{4}$ was employed in order to achieve an adequate amplification of strept-AuNPs present on sensor surface to allow their direct observation by SEM. SEM images taken at an acceleration voltage of $3 \mathrm{kV}$ are shown in Figure 4, illustrating a positive experiment with sandwich protocol and strep-AuNPs conjugation. As can be observed in Figure 4a), the distribution of gold enhanced-gold nanoparticles is quite homogeneous. This also implies a regular distribution of MWCNT and well-organized formation of sandwich complex onto the electrode surface. This high density distribution also demonstrates the proper functionality of the MWCNT platform and the immobilization of the biomolecule. Comparing this experiment with the negative control that did not use the biotinylated AbCytc, Figure 4b), a surface with almost absent nanoparticles can be observed. 


\section{Analytical performance of the aptamer-antibody sandwich assay for detection of Cytc}

\section{<TABLE 1>}

After experimental concentration optimization, the proposed aptasensor was then used following the sandwich protocol, plus amplification employing the strep-AuNPs and silver enhancement treatment. Figure 5 shows calibration curves with increasing concentrations of Cytc and their respective regression lines in the logaritmic scale, at the different steps of the protocol: (1) AptCytc-Cytc, (2) Sandwich formation between AptCytc, Cytc and AbCytc, (3) aptamer sandwich modified with strep-AuNPs, and (4) aptamer sandwich modified with strep-AuNPs and silver enhancement treatment. Although the reproducibilities were not ideal in all cases, the calibration curves obtained showed a good RSD. As can be seen in Figure 5, all calibration curves increased until the value of $100 \mathrm{pM}$ of Cytc, this could be due to the fact that concentrations larger than $100 \mathrm{pM}$ cause a saturation on the sensor surface. As can be observed in Table 1, the use of silver enhancement treatment led to the highest sensitivity and signal amplification, $117 \%$, compared to the simple biosensing scheme. This demostrates that the silver deposition on gold nanoparticles, basically increases the sterical hindrance, producing an increment of observed impedance, given this conductive silver is not wired to the electrode surface. Despite a higher sensitivity, the detection limit obtained in this case, $1.9 \mathrm{pM}$, is slightly worse than the one obtained with only strep-AuNPs. This fact could be due to the increased number of process steps and associated increased potential errors. The best detection limit, approximately $1.5 \mathrm{pM}$, corresponded to the sandwich and strep-AuNPS calibration curve. This confirmed that the proposed methods show a low detection limit and an ultrahigh sensitivity for the detection of Cytc.

\section{$<$ FIGURE 5>}

\section{Selectivity of the aptamer-antibody sandwich assay}

Control experiments were conducted to investigate the specificity of aptamerantibody assay. In this work, majority serum proteins, such as human IgG, fibrinogen and albumin at serum physiological levels were tested to operate the proposed aptasensor instead of Cytc under the same experimental conditions. As can be seen in Figure 6, the presence of interfering proteins such as albumin, fibrinogen and 
immunoglobulin $\mathrm{G}$, at serum concentration level exhibits negligible response compared with 100pM Cytc in the amplified sandwich protocol, even at concentrations four or five orders of magnitude higher than typical Cytc concentrations. Figure 6 also demonstrates that the sandwich protocol displays as clear advantage, more than the signal amplification, the marked decrease of interfering effects that are still remarkable in the simple biosensing protocol. This is specially remarkable for IgG protein (human), which shows appreciable interference in the assay without amplification, although it becomes practically negligible with the sandwich variant.

<FIGURE 6>

\section{Conclusions}

An ultrasensitive aptamer-antibody sandwich assay for cytochrome c detection using electrochemical impedance technique was reported. Due to the signal amplification with strep-AuNPs and silver deposition, it was possible to increase the sensitivity of the assay. Additionally, for a comparable amount of AptCytc-Cytc, the signal resulted in a $117 \%$ amplification, compared to results recorded with simple biosensing AptCytc-Cytc. Furthermore, the limit of detection obtained was $1.9 \mathrm{pM}$. A good linear range, 25-100pM, and high selectivity with respect to different serum proteins at serum concentration level were also achieved with this protocol thanks to the double recognition scheme utilized. Finally, the gold enhancement treatment permitted the visualization of the gold nanoparticles on the electrode surface with SEM.

\section{Acknowledgments}

This research was partly supported by the Research Executive Agency (REA) of the European Union under Grant Agreement number PITN-GA-2010-264772 (ITN CHEBANA), by the Ministry of Science and Innovation (MCINN, Madrid, Spain) through the project CTQ2010-17099 and by the Catalonia program ICREA Academia. Cristina Ocaña thanks the support of Ministry of Science and Innovation (MICINN, Madrid, Spain) for the predoctoral grant. 


\section{References}

1. Alleyne T, Joseph J, Sampson V (2001) Cytochrome-c detection: A diagnostic marker for myocardial infarction. Applied Biochemistry and Biotechnology 90 (2):97105.

2. Clark SL, Remcho VT (2002) Aptamers as analytical reagents. Electrophoresis 23 (9):1335-1340.

3. Radi A, Acero Sánchez JL, Baldrich E, O'Sullivan CK (2005) Reusable Impedimetric Aptasensor. Anal Chem 77 (19):6320-6323.

4. Biesecker G, Dihel L, Enney K, Bendele RA (1999) Derivation of RNA aptamer inhibitors of human complement C5. Immunopharmacology 42 (1-3):219-230.

5. Hicke BJ, Marion C, Chang YF, Gould T, Lynott CK, Parma D, Schmidt PG, Warren S (2001) Tenascin-C aptamers are generated using tumor cells and purified protein. J Biol Chem 276 (52):48644-48654.

6. Cox JC, Ellington AD (2001) Automated selection of anti-protein aptamers. Bioorganic \& medicinal chemistry 9 (10):2525-2531

7. Cai H, Lee TM-H, Hsing IM (2006) Label-free protein recognition using an aptamerbased impedance measurement assay. Sensors and Actuators B: Chemical 114 (1):433437.

8. Jayasena SD (1999) Aptamers: an emerging class of molecules that rival antibodies in diagnostics. Clin Chem 45 (9):1628-1650

9. Ellington AD, Szostak JW (1990) In vitro selection of RNA molecules that bind specific ligands. Nature 346 (6287):818-822

10. Tuerk C, Gold L (1990) Systemic evolution of ligands by exponential enrichment: RNA ligands to bacteriophage T4 DNA polymerase. Science 249 (4968):505-510

11. Tombelli S, Minunni M, Mascini M (2005) Analytical applications of aptamers. Biosens Bioelectron 20 (12):2424-2434

12. Zhang L, Cui P, Zhang B, Gao F (2013) Aptamer-based turn-on detection of thrombin in biological fluids based on rfficient phosphorescence energy transfer from Mn-doped ZnS quantum dots to carbon nanodots. Chemistry - A European Journal 19 (28):9242-9250

13. Chang M, Kwon M, Kim S, Yunn NO, Kim D, Ryu SH, Lee JB (2014) Aptamerbased single-molecule imaging of insulin receptors in living cells. Journal of Biomedical Optics 19 (5)

14. Loo AH, Bonanni A, Pumera M (2012) Impedimetric thrombin aptasensor based on chemically modified graphenes. Nanoscale 4 (1):143-147

15. Ho MY, D'Souza N, Migliorato P (2012) Electrochemical Aptamer-Based Sandwich Assays for the Detection of Explosives. Anal Chem 84 (10):4245-4247.

16. Lee M, Walt DR (2000) A fiber-optic microarray biosensor using aptamers as receptors. Anal Biochem 282 (1):142-146. doi:10.1006/abio.2000.4595

17. Mairal T, Nadal P, Svobodova M, O'Sullivan CK (2014) FRET-based dimeric aptamer probe for selective and sensitive Lup an 1 allergen detection. Biosens Bioelectron 54:207-210

18. Srijanto BR, Cheney CP, Hedden DL, Gehl AC, Crilly PB, Huestis MA, Ferrell TL (2012) Piezoresistive Microcantilevers-Based Cocaine Biosensors. Sens Lett 10 (34):850-855. 
19. Collins CM, Yui S, Roberts CES, Kojic I (2013) Thrombin detection using a piezoelectric aptamer-linked immunosorbent assay. Anal Biochem 443 (1):97-103

20. Ocaña C, del Valle M (2013) A comparison of four protocols for the immobilization of an aptamer on graphite composite electrodes. Microchim Acta:1-9

21. Kara P, de la Escosura-Muñiz A, Maltez-da Costa M, Guix M, Ozsoz M, Merkoçi A (2010) Aptamers based electrochemical biosensor for protein detection using carbon nanotubes platforms. Biosens Bioelectron 26 (4):1715-1718

22. McDonald JR (1987) Impedance Spectroscopy. John Wiley, New York

23. Bonanni A, Ambrosi A, Pumera M (2012) On Oxygen-Containing Groups in Chemically Modified Graphenes. Chem-Eur J 18 (15):4541-4548.

24. Bonanni A, Ambrosi A, Pumera M (2012) Nucleic Acid Functionalized Graphene for Biosensing. Chem-Eur J 18 (6):1668-1673.

25. Bardea A, Patolsky F, Dagan A, Willner I (1999) Sensing and amplification of oligonucleotide-DNA interactions by means of impedance spectroscopy: a route to a Tay-Sachs sensor. Chem Comm (1):21-22.

26. Loo AH, Bonanni A, Ambrosi A, Poh HL, Pumera M (2012) Impedimetric immunoglobulin $\mathrm{G}$ immunosensor based on chemically modified graphenes. Nanoscale 4 (3):921-925.

27. Moreno-Guzmán M, Ojeda I, Villalonga R, González-Cortés A, Yáñez-Sedeño P, Pingarrón JM (2012) Ultrasensitive detection of adrenocorticotropin hormone (ACTH) using disposable phenylboronic-modified electrochemical immunosensors. Biosens Bioelectron 35 (1):82-86.

28. Zhang Y, Li Y, Wu W, Jiang Y, Hu B (2014) Chitosan coated on the layers' glucose oxidase immobilized on cysteamine/Au electrode for use as glucose biosensor. Biosens Bioelectron 60:271-276

29. Bonanni A, Esplandiu MJ, del Valle M (2010) Impedimetric genosensing of DNA polymorphism correlated to cystic fibrosis: A comparison among different protocols and electrode surfaces. Biosens Bioelectron 26 (4):1245-1251.

30. Bonanni A, Pumera M, Miyahara Y (2010) Rapid, Sensitive, and Label-Free Impedimetric Detection of a Single-Nucleotide Polymorphism Correlated to Kidney Disease. Anal Chem 82 (9):3772-3779.

31. Blin F, Koutsoukos P, Klepetsianis P, Forsyth M (2007) The corrosion inhibition mechanism of new rare earth cinnamate compounds - Electrochemical studies. Electrochim Acta 52 (21):6212-6220.

32. Liao Y-M, Feng Z-D, Chen Z-L (2007) In situ tracing the process of human enamel demineralization by electrochemical impedance spectroscopy (EIS). Journal of Dentistry 35 (5):425-430.

33. Bonanni A, del Valle M (2010) Use of nanomaterials for impedimetric DNA sensors: A review. Anal Chim Acta 678 (1):7-17.

34. Deng C, Chen J, Nie Z, Wang M, Chu X, Chen X, Xiao X, Lei C, Yao S (2008) Impedimetric Aptasensor with Femtomolar Sensitivity Based on the Enlargement of Surface-Charged Gold Nanoparticles. Anal Chem 81 (2):739-745.

35. Zheng J, Feng W, Lin L, Zhang F, Cheng G, He P, Fang Y (2007) A new amplification strategy for ultrasensitive electrochemical aptasensor with network-like thiocyanuric acid/gold nanoparticles. Biosens Bioelectron 23 (3):341-347.

36. Bonanni A, Esplandiu MJ, del Valle M (2008) Signal amplification for impedimetric genosensing using gold-streptavidin nanoparticles. Electrochim Acta 53 (11):4022-4029. 
37. Bonanni A, Esplandiu MJ, Pividori MI, Alegret S, del Valle M (2006) Impedimetric genosensors for the detection of DNA hybridization. Anal Bioanal Chem 385 (7):11951201.

38. Cai H, Wang YQ, He PG, Fang YH (2002) Electrochemical detection of DNA hybridization based on silver-enhanced gold nanoparticle label. Anal Chim Acta 469 (2):165-172.

39. Hanaee H, Ghourchian H, Ziaee AA (2007) Nanoparticle-based electrochemical detection of hepatitis B virus using stripping chronopotentiometry. Anal Biochem 370 (2):195-200. 


\section{FIGURE CAPTIONS}

Fig.1 Scheme of the experimental protocol.

Fig.2 a) Nyquist diagrams of: $(\bullet)$ Bare electrode, (०) Electrochemical grafting treatment and ( $\mathbf{\nabla})$ Aptamer immobilization. b) Nyquist diagrams of: $(\bullet)$ Bare electrode, ( $($ ) aptamer immobilization, ( $\mathbf{\nabla})$ Aptamer with Cytc, $(\Delta)$ sandwich complex with antibody, (-) sandwich complex modified with goldnanoparticles and (口) sandwich complex modified with gold-nanoparticles and silver enhancement treatment. All experiments were performed in PBS solution and all EIS measurements were performed in PBS solution containing $0.01 \mathrm{M} \mathrm{K}_{3}\left[\mathrm{Fe}(\mathrm{CN})_{6}\right] / \mathrm{K}_{4}\left[\mathrm{Fe}(\mathrm{CN})_{6}\right]$.

Fig. 3 a) Optimization of the amount of cytochrome $c$ aptamer AptCytc immobilized on each electrode. b) Optimization of the concentration of the blocking agent, PEG. Uncertainty values corresponding to replicated experiments $(\mathrm{n}=5)$.

Fig.4 SEM images of (a) experiment using sanwich complex + strep-AuNPS+ gold enhancement treatment (b) negative control using Aptcytc + cytochrome $\mathrm{c}+$ non complementary aptamer + strepAuNPs + gold enhancement treatment. All images were taken at an acceleration voltage of $3 \mathrm{kV}$ and a resolution of $2 \mu \mathrm{m}$.

Fig.5 Calibration and regression curves of: (1) (black circle) AptCytc and Cytc, (2) (white circle) sandwich complex, (3) (black triangle) sandwich complex modified with strep-AuNPs, (4) (white triangle) sandwich complex modified with strep-AuNPs and silver enhancement treatment. All experiments were performed in PBS solution and all EIS measurements were performed in PBS solution containing $0.01 \mathrm{M} \mathrm{K} \mathrm{K}_{3}\left[\mathrm{Fe}(\mathrm{CN})_{6}\right] / \mathrm{K}_{4}\left[\mathrm{Fe}(\mathrm{CN})_{6}\right]$. Uncertainty values corresponding to replicated experiments $(\mathrm{n}=5)$.

Fig.6 3D bar chart of responses towards different proteins present in serum, with simple biosensing scheme and with the sandwich/amplification protocol. Uncertainty values corresponding to replicated experiments $(\mathrm{n}=5)$. 

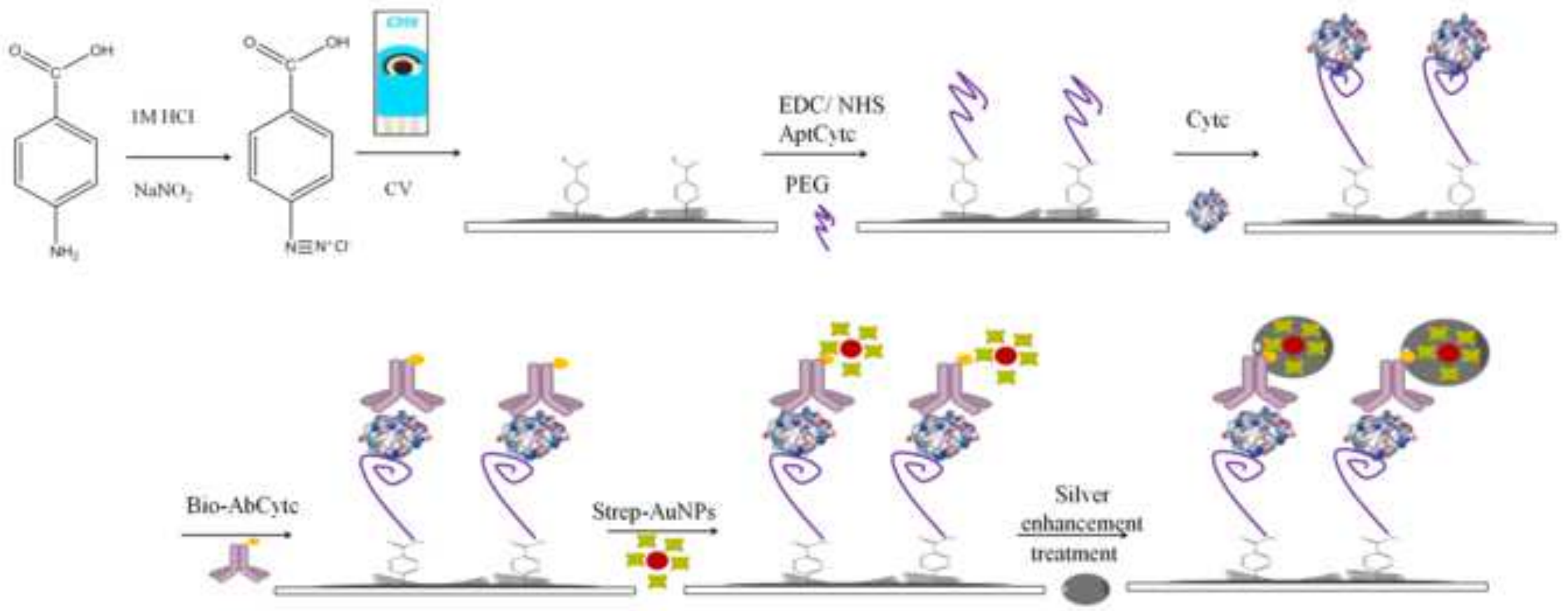

PEG Polvetwy lenczlyeas 

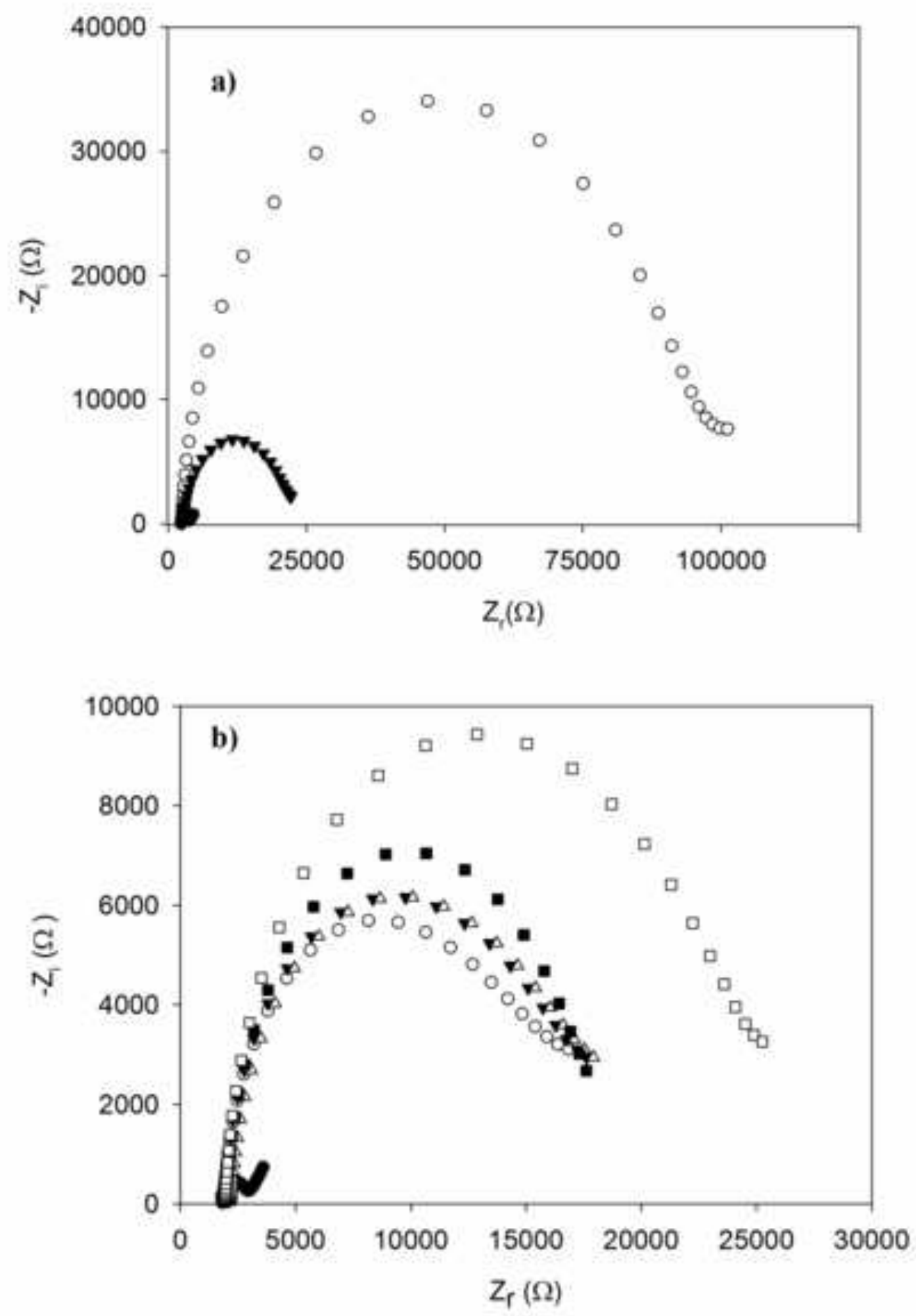

Figure 2 

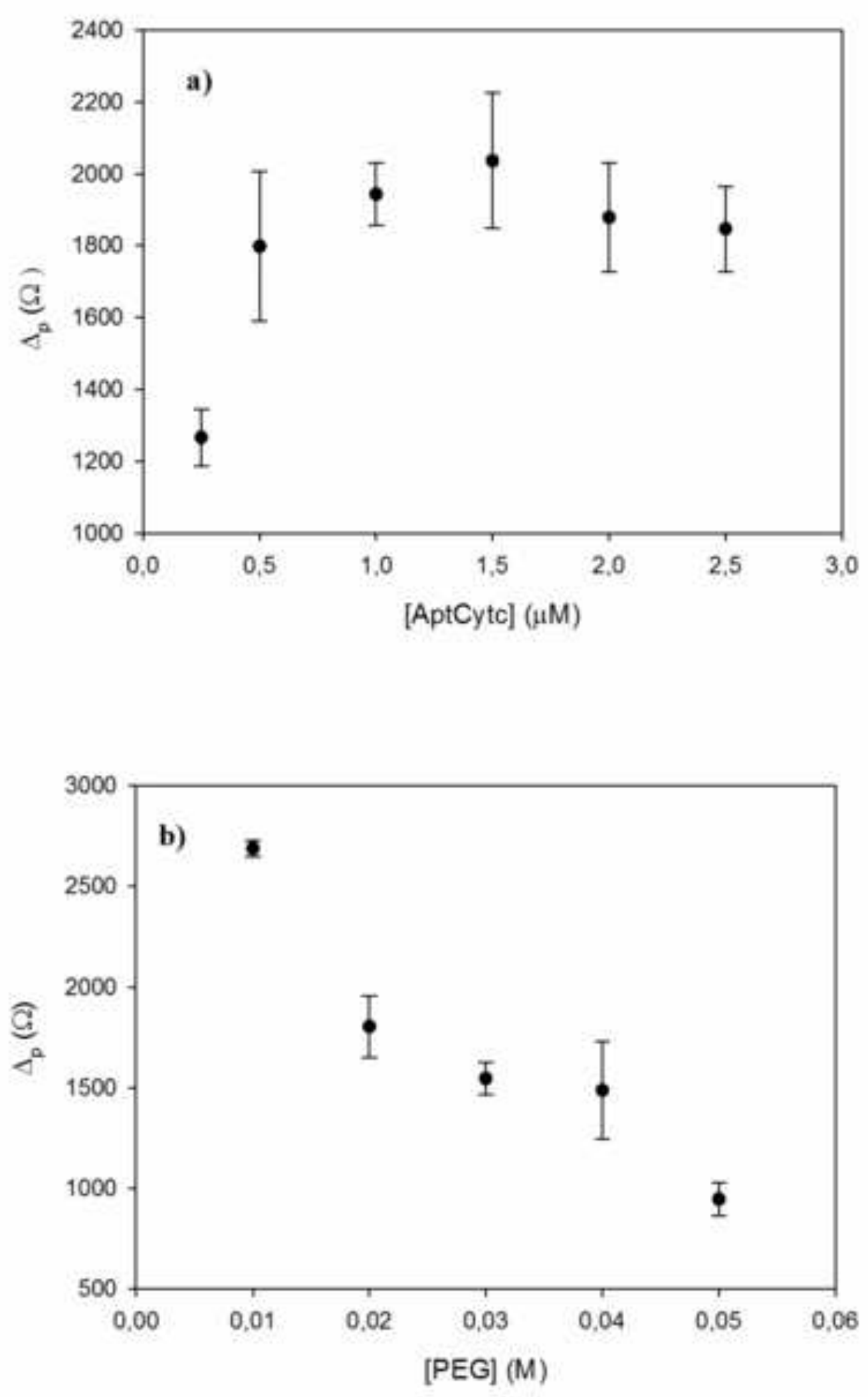

Figure 3

\section{Figure 3}


Figure 4
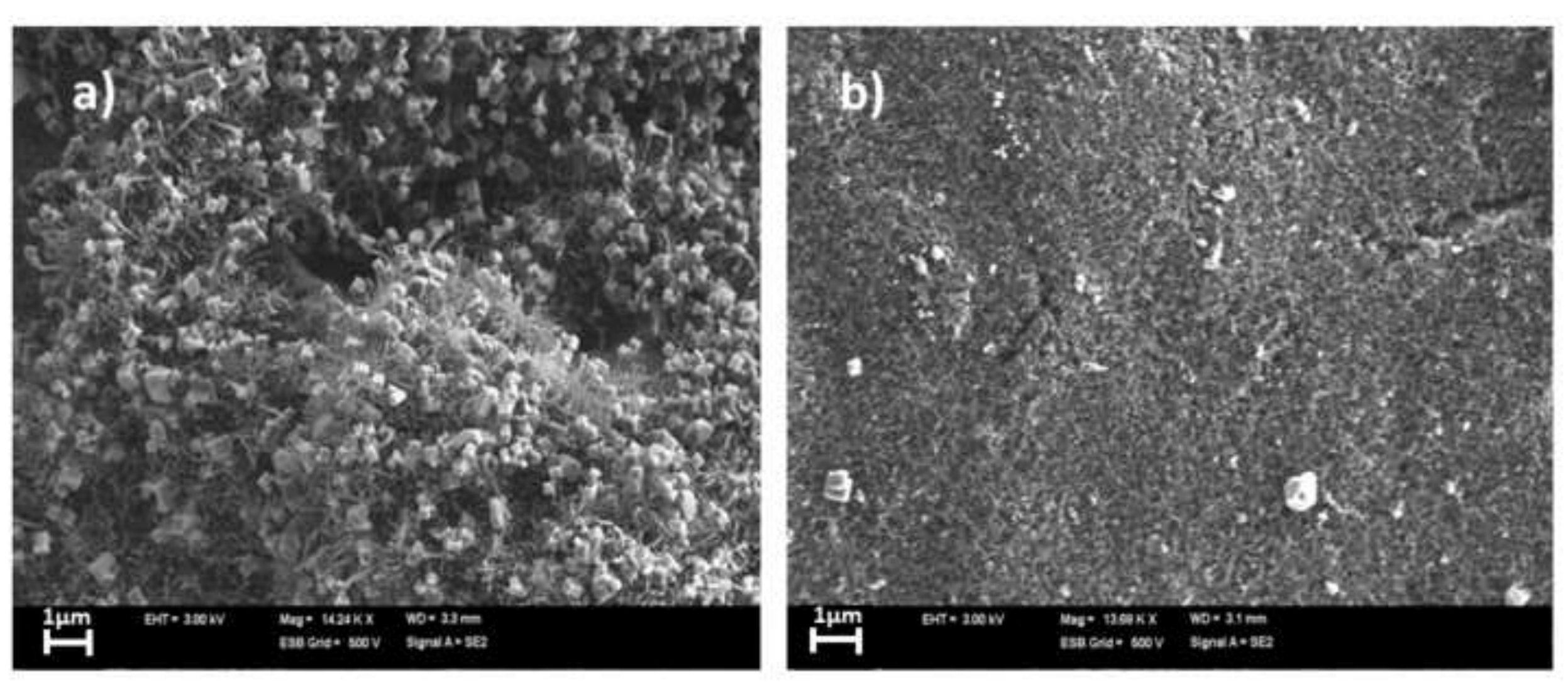


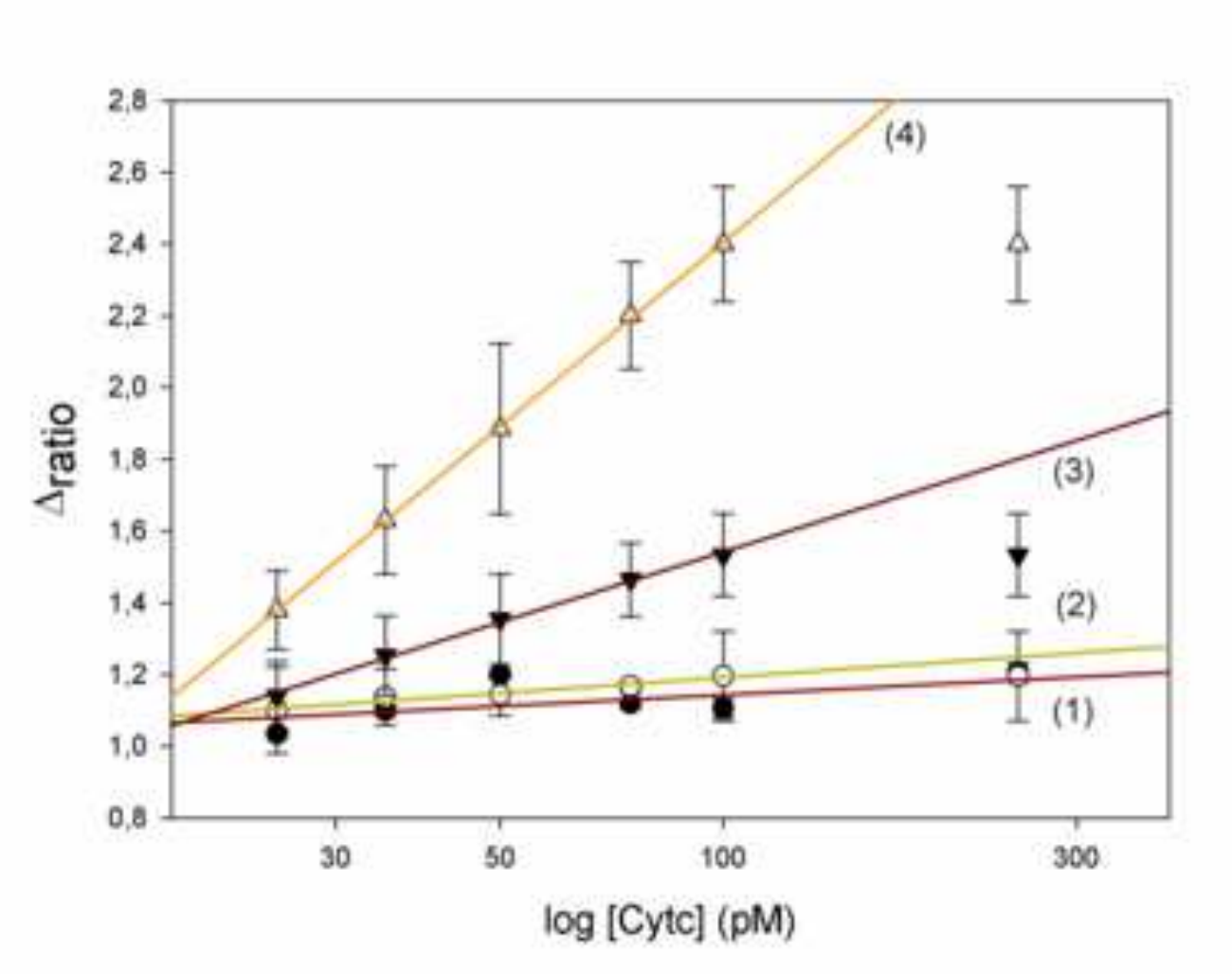

Figure 5

Figure 5

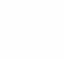




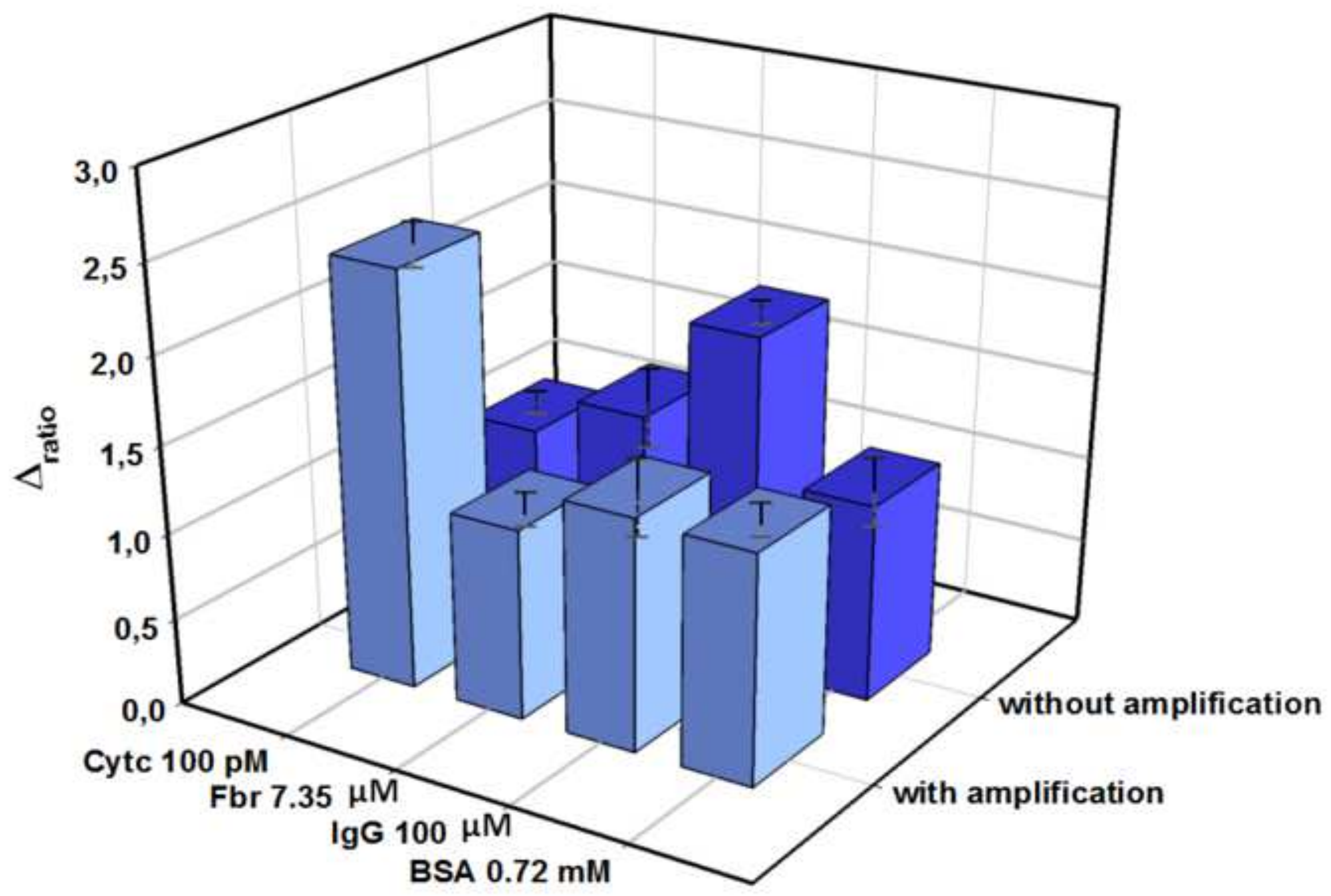


Table 1. Summary of the different calibration curves considering different stages of the assay.

\begin{tabular}{|c|c|c|c|c|}
\hline Calibration curve & Regression curve & Detection limit (pM) & Net Amplification \% & RSD $^{*} \%$ \\
\hline (1) AptCytc/ Cytc & $\Delta$ ratio $=0.852+0.058 \cdot \log [\mathrm{Cyt} c]$ & 25 & - & 2.3 \\
\hline (2)Sandwich complex & $\Delta$ ratio $=0.882+0.673 \cdot \log [$ Cytc $]$ & 13 & 8 & 3.2 \\
\hline (3) Sandwich/strep-AuNPs & $\Delta$ ratio $=0.226+0.286 \cdot \log [\mathrm{Cyt} c]$ & 1.5 & 40 & 5.9 \\
\hline (4) Sandwich/strep-AuNPs/ & $\Delta$ ratio $=-1.00+0.739 \cdot \log [$ Cytc $]$ & 1.9 & 117 & 6.8 \\
\hline Silver enhanc. & & & & \\
\hline
\end{tabular}

${ }^{*}$ Corresponding to five replicated experiments at $0.75 \mathrm{pM}$ 